\title{
Linx
}

Revue des linguistes de l'université Paris X Nanterre

38 | 1998

L'acquisition de la temporalité en situation bilingue

\section{Phénomènes de contact de langues dans le parler bilingue fongbe-français}

Language contact phenomena in Fongbe-French bilingual discourse

\section{Comlan-Zéphirin Tossa}

\section{(2) OpenEdition}

\section{Journals}

Édition électronique

URL : http://journals.openedition.org/linx/938

DOI : $10.4000 /$ linx.938

ISSN : 2118-9692

\section{Éditeur}

Presses universitaires de Paris Nanterre

\section{Édition imprimée}

Date de publication : 1 décembre 1998

Pagination : 197-220

ISSN : 0246-8743

\section{Référence électronique}

Comlan-Zéphirin Tossa, «Phénomènes de contact de langues dans le parler bilingue fongbe-

français », Linx [En ligne], 38 | 1998, mis en ligne le 02 juillet 2012, consulté le 30 avril 2019. URL :

http://journals.openedition.org/linx/938 ; DOI : 10.4000/linx.938

Ce document a été généré automatiquement le 30 avril 2019.

Département de Sciences du langage, Université Paris Ouest 


\title{
Phénomènes de contact de langues dans le parler bilingue fongbe-français
}

\author{
Language contact phenomena in Fongbe-French bilingual discourse
}

\author{
Comlan-Zéphirin Tossa
}

\section{Introduction 1}

1 Le fongbe est une langue du groupe kwa de la famille Niger-Congo. Il fait partie d'un sousgroupe, appelé le sous-groupe gbe, qui compte une trentaine de langues. Le fongbe est une langue béninoise parlée notamment dans la ville de Cotonou, la capitale économique du Bénin.

2 Le Bénin est un pays où plusieurs langues nationales coexistent avec le français dans une situation de diglossie. Le français y joue un rôle prépondérant. C'est en particulier dans cette langue qu'est faite l'éducation scolaire et tout Béninois ayant atteint un certain niveau de scolarisation (par exemple la classe de troisième de l'enseignement secondaire) a une maîtrise appréciable du français et, à ce titre, peut être considéré comme bilingue.

Quand on écoute parler les bilingues fongbe-français, surtout dans les villes, et en particulier à Cotonou, on relève trois modes essentiels de communication langagière : le fongbe, le français, et une sorte de mélange de ces deux langues. La présente étude se propose d'analyser ce discours bilingue en dégageant les caractéristiques formelles de certains phénomènes de contact qui y interviennent.

4 L'étude est organisée en quatre parties. La première présente le cadre théorique dans lequel s'insèrent les analyses. Dans une deuxième partie, nous étudions le phénomène d'alternance ou code-switching, après avoir présenté les données. La troisième partie est consacrée à l'étude des phénomènes d'emprunt. L'étude se termine par la présentation du phénomène d'insertion de constituant et par l'analyse d'un cas particulier de ce phénomène dans le discours bilingue fongbe-français. 


\section{Le cadre théorique}

5 Le problème fondamental qui se pose quand on étudie des phénomènes de contact de langues est de déterminer la nature des faits observés. Les divergences conceptuelles et terminologiques rencontrées dans la littérature exigent la délimitation d'un cadre théorique précis pour maintenir une certaine cohésion.

6 Le cadre théorique dans lequel sera menée cette étude est celui dit de la variation (cf. entre autres Labov 1969, 1971, 1984; G. Sankoff 1974; Poplack 1980, 1988, 1993 ; D. Sankoff 1982,1988$)$. Le modèle variationniste est l'un de ceux qui se sont développés ces dernières années dans le domaine de l'étude des discours de bilingues. Les tenants de ce modèle prônent l'utilisation du discours ordinaire quotidien et informel pour démontrer que les faits de mélange de langues présentent une régularité qui transcende leur instabilité apparente. Poplack (1988: 24) cite les principes suivants qui sont à la base du modèle :

- “1. l'utilisation de données appropriées, c'est-à-dire l'étude du langage dans son contexte naturel,

- 2. l'analyse de toutes les données pertinentes (et pas seulement celles qui appuient notre théorie),

- 3. le choix des informateurs de façon à assurer leur représentativité, et la connaissance de ce qu'ils représentent, et

- 4. la délimitation du contexte variable, c'est-à-dire la définition de l'objet de l'étude".

7 Les chercheurs travaillant dans le modèle variationniste font porter leurs analyses sur de larges échantillons de discours pour vérifier la régularité des structures, tout en effectuant des analyses quantitatives fondées sur des calculs de type statistique. Ces calculs portent sur les fréquences d'occurrences de faits de contact de langues étudiés, et doivent corroborer les résultats trouvés par l'analyse grammaticale. Tel est le cadre global dans lequel les variationnistes mènent leur travaux dans le domaine de l'alternance de langues. Une fois ce cadre établi, on a eu besoin de mettre au point des outils formels pour analyser les discours et établir des distinctions entre les divers phénomènes qu'on rencontre dans les mélanges de langues. Ces outils concernent essentiellement des concepts et des règles ou contraintes.

8 Un nombre important de règles et de contraintes ont été proposées dans la littérature pour rendre compte des structures rencontrées dans les discours des bilingues (cf. par exemple Pfaff 1979). Mais dans le souci de rendre le modèle opérationnel et plus maniable Poplack $(1980,1981)$ a réduit les règles à deux contraintes fondamentales qui, prises ensembles, paraissent assez générales pour permettre de faire la distinction au niveau de la plupart des phénomènes de contact de langues. Ces deux contraintes constituent aujourd'hui la base de ce qu'on appelle la "grammaire du code-switching" (cf. Sankoff \& Poplack 1981). La première contrainte est celle dite du morphème libre. Elle a été reprécisée par Poplack (1982:12) et stipule qu'une alternance est interdite entre un morphème lié et une forme lexicale à moins que cette dernière soit phonologiquement intégrée dans la langue du morphème. Nous verrons que cette contrainte intervient dans les données bilingues fongbe-français.

9 La seconde contrainte est la contrainte de l'équivalence. Elle prédit que l'alternance entre deux langues "peut se produire librement entre deux éléments quelconques d'une phrase, 
pourvu qu'ils soient ordonnés de la même façon selon les règles de leurs grammaires respectives" (Poplack 1988: 23). Une illustration de cette contrainte a été donnée par Poplack (1980 : 586) avec le schéma en (1) ci-dessous.

(1)

(1)

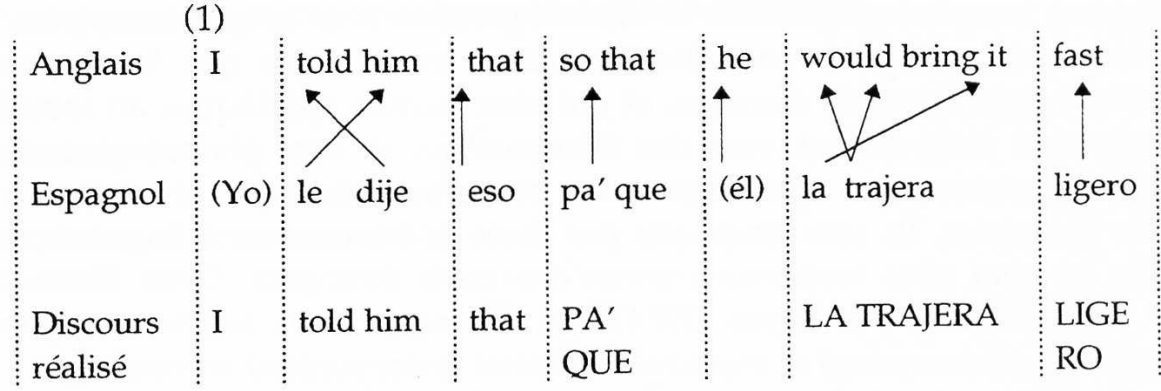
l'emprunt. L'alternance se définit comme "des fragments de phrases provenant d'une langue pourvus des caractéristiques morphologiques, syntaxiques et lexicales propres à cette langue, et qui viennent se juxtaposer à un fragment d'une autre langue" (Poplack 1988 :36). L'emprunt par contre implique des éléments d'une langue L2 introduits dans une langue réceptrice L1, mais subissant "des adaptations les rendant conformes aux patrons phonologiques, morphologiques et syntaxiques de la langue réceptrice." (Poplack 1989 : 142).

On distingue cependant les emprunts spontanés des emprunts intégrés. Les emprunts spontanés gardent la structure phonologique de la langue L2 mais s'intègrent morphologiquement et syntaxiquement à la langue réceptrice L1. Les emprunts spontanés sont des emprunts occasionnels que les locuteurs effectuent dans l'acte de 
discours, et qui sont parfois spécifiques au locuteur. Les emprunts intégrés sont, eux, des éléments qui se sont phonologiquement, morphologiquement et syntaxiquement conformés de façon évolutive à la langue réceptrice. Ils sont employés par toute la communauté linguistique et parfois ne sont plus reconnus comme des mots étrangers. Cette distinction avait déjà été faite par Reyes (1974), qui distingue entre emprunts spontanés (spontaneous borrowing) et emprunts incorporés (incorporated borrowing).

Les alternances elles-mêmes sont réparties en deux types fondamentaux: l'alternance fluide et l'alternance balisée. Une alternance est dite fluide quand le passage d'une langue à l'autre s'effectue sans heurt ni transition. Comme le dit Poplack (1988), l'alternance fluide n'est ni précédée, ni suivie de pause ou d'hésitation. Elle n'est pas une traduction ni une répétition de ce qui précède dans l'énoncé. On n'y observe aucun effet rhétorique. Le locuteur n'y attire pas l'attention de l'auditeur et ce dernier n'est pas obligé de la ratifier. L'alternance est dite balisée quand, au contraire, elle est marquée par des stratégies discursives comme l'hésitation, la recherche du mot juste, le commentaire métalinguistique, l'identification de l'appartenance linguistique du morceau de phrase, la répétition ou la traduction. Dans une alternance balisée, le locuteur peut attirer l'attention de l'auditeur sur l'alternance en la rendant saillante.

Les alternances fluides sont soit intra-phrastiques soit inter-phrastiques, alors que les alternances balisées sont en général extra-phrastiques. L'alternance intra-phrastique s'effectue à l'intérieur d'une proposition ou d'une phrase et est fortement soumise aux contraintes syntaxiques. L'alternance inter-phrastique s'effectue entre deux propositions ou entre deux phrases. Il n'est pas toujours facile de distinguer l'alternance interphrastique et l'alternance extra-phrastique. Cependant, comme l'indique Silvestri (1996: 192)), l'alternance extra-phrastique "comporte des éléments ayant des fonctions autres que syntaxiques; ils sont faciles à introduire dans le discours parce que ce sont de simples ajouts." C'est le cas des "tags", des mots ou expressions emblématiques, qui ont de fortes connotations ethniques et qui sont difficiles à traduire. Silvestri ajoute qu'il y a "aussi les "fillers" ou mots du discours (c'est-à-dire des formules figées), similaires aux tags, par exemples "that's all", "you know", "I know". Les interjections, les expressions idiomatiques et les citations sont aussi des alternances extra-phrastiques.

Voilà brièvement présenté le cadre théorique dans lequel les faits de contact de langues sont analysés dans la présente étude.

En ce qui concerne les descriptions syntaxiques, nous nous fondons sur la théorie Xbarre. Elle fait partie du modèle Gouvernement et Liage de la Grammaire Générative et postule que les structures syntaxiques des langues naturelles s'organisent selon trois niveaux de projection qui sont $\mathrm{X}^{\circ}, \mathrm{X}^{\prime}, \mathrm{XP} . \mathrm{X}^{\circ}$ est une tête c'est-à-dire un constituant ultime. $X^{\prime}$ est obtenu par la projection de $X^{\circ}$ quand ce dernier prend un complément. Mais $X^{\prime}$ peut se projeter de façon récursive en un deuxième $X^{\prime}$ en prenant un adjoint. $X$ ' se projette ensuite en XP en prenant un spécifieur. XP est une projection maximale. La structure générale d'une phrase est donnée par la représentation suivante :

(2)

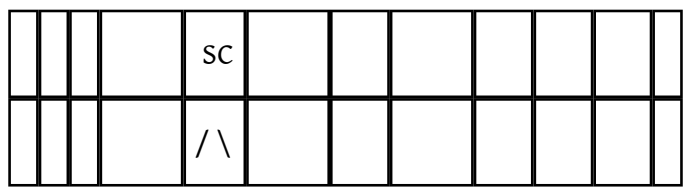




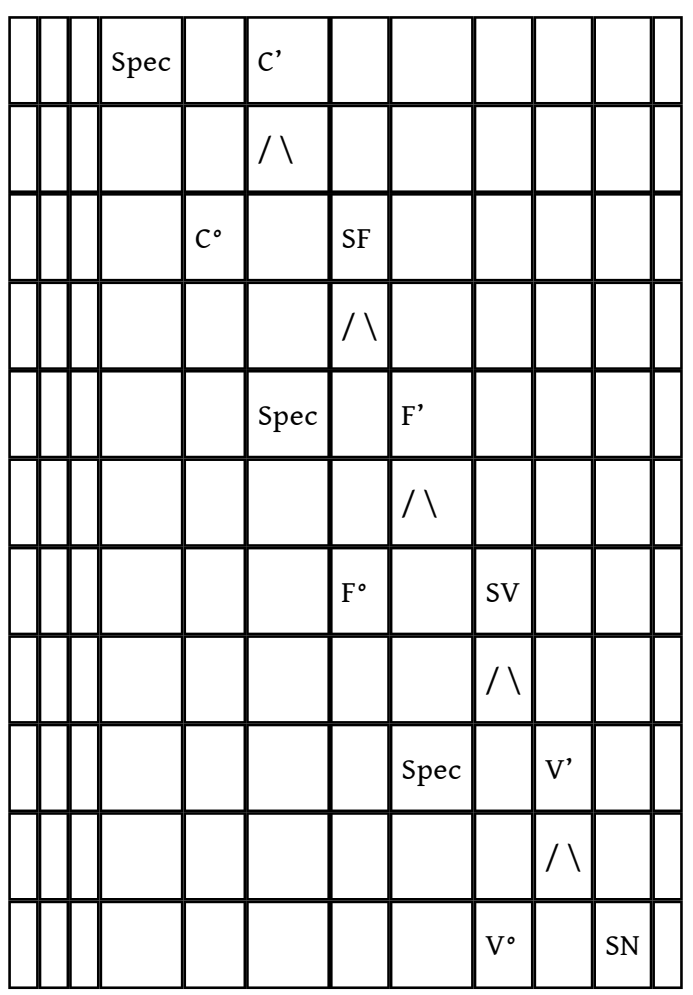

Dans (2), SC représente la proposition ou syntagme de "complémentation», et C représente un "complémenteur" ou plus précisément un subordonnant. SF signifie syntagme fonctionnel et $\mathrm{F}$ représente la flexion, c'est-à-dire les marqueurs du verbe tels que le temps, l'aspect et le mode. SV est le syntagme verbal et SN le syntagme nominal.

\section{Les données}

21 Les analyses proposées ici s'appuient sur un corpus oral recueilli essentiellement dans la ville de Cotonou, auprès de personnes bilingues fongbe-français. Tous sont de langue maternelle fongbe mais ont un niveau de scolarisation en français atteignant au moins la classe de troisième de l'enseignement secondaire. Nous avons recueilli, sur six cassettes d'une heure, des productions langagières dans des conditions propres à l'émergence de discours spontané obtenu en réaction à des thèmes comme la situation économique difficile, l'exode des jeunes dans les pays voisins, la recherche d'emploi, etc. L'enquête proprement dite a été faite avec l'aide d'étudiants en maîtrise de linguistique qui prétendaient faire des visites de courtoisie à des parents ou amis. Nous avons aussi effectué un enregistrement d'une cassette à Montréal auprès d'étudiants de troisième cycle de langue maternelle fongbe.

22 Les cassettes ont ensuite été transcrites. L'ensemble du corpus a été saisi sur ordinateur et traité par un logiciel qui a permis de dégager les concordances de tous les mots. Les concordances permettent de faire l'inventaire de tous les mots du corpus et d'observer les distributions, c'est-à-dire l'environnement à gauche et à droite de chaque mot.

23 A cet ensemble de données nous avons ajouté quelques faits que nous avons observés fortuitement au cours de diverses conversations. 


\section{Alternances fluides et alternances balisées en fongbe-français}

La distinction entre "vraies" alternances et emprunts étant établie, il est frappant de noter que dans le discours bilingue fongbe-français, on relève beaucoup plus de cas d'emprunt que d'alternance, ce qui n'est en fait pas surprenant, le fongbe et le français étant deux langues typologiquement différentes. En effet, dans divers travaux entrepris sur des couples de langues typologiquement différents (cf. Sankoff et al. (1986), Poplack et al. (1987), Poplack (1988)) il a été montré que les types de mélange de langues les plus fréquents sont les emprunts quand les langues en contact présentent des différences typologiques syntaxiques. C'est le cas entre le fongbe et le français.

L'étude des alternances de langues ou code-switching peut porter sur plusieurs aspects, et les raisons qui motivent le passage d'une langue à une autre sont multiples. Gobbi (1990) a mené une étude sur le choix de langue dans une situation de diglossie entre un dialecte de Lombardie et l'italien standard. Les raisons socio-psychologiques qui justifient le choix dans les cas d'alternance ont été identifiées dans cette étude. Dans un rapport fait par Titone (194: 69-70) sur le travail de Gobbi, on note 17 raisons différentes justifiant le choix de la langue dite faible, et 12 raisons motivant le choix de la variété dite forte.

Dans la présente étude nous ne nous sommes pas particulièrement intéressé à ces aspects extralinguistiques. Nous avons observé globalement les faits afin de caractériser les alternances du point de vue formel.

\subsection{Alternances fluides en fongbe-français}

En s'appuyant sur la définition de l'alternance fluide donnée plus haut, on observe que dans le discours du bilingue fongbe-français, le passage d'une langue à l'autre se fait plus fréquemment selon cette forme d'alternance. Les locuteurs fongbe-français n'attirent généralement pas l'attention de l'auditeur sur le changement de langue.

\subsubsection{Alternances intra-phrastiques}

La contrainte d'équivalence prédit l'absence d'alternances entre des constituants de deux langues ordonnés de façon différente dans ces deux langues, mais prédit également l'alternance en des endroits où on a le même ordre pour les constituants. Le fongbe et le français ont en partage l'ordre SVO. On doit donc s'attendre à des alternances entre le syntagme nominal sujet et le prédicat puis entre le verbe et le syntagme nominal objet. Cependant on trouve très peu de vraies alternances entre ces différents constituants. La raison en est qu'au lieu d'avoir des alterances on a, la plupart du temps, des emprunts. Les alternances intra-phrastiques qu'on trouve en fongbe-français se situent généralement à des points où on ne trouve pas d'emprunt spontané, par exemple entre le verbe et le syntagme propositionnel, comme dans (3) ci-dessus :

\begin{tabular}{|l|l|l|l|l|l|l|}
\hline$(3)$ & a. & á & prendre & tòn & sur le vif & \\
\hline & & tu & prendre & & le sur le vif & \\
\hline
\end{tabular}




\begin{tabular}{|l|l|l|l|l|l|l|}
\hline & & 'tu l'a & pris sur & le vif' & & \\
\hline & & & & & & \\
\hline & b. & à & dj̀ & à un moment donné & dò mè & bì yì \\
\hline \hline & & & & & que monde & tout partir \\
\hline & & 'tu as & dit & à un moment donné & que tout le monde & est parti' \\
\hline & & & & & & \\
\hline & c. & dahwé & ó & yì & Guinée & pendant trois semaines \\
\hline \hline & & homme & DEF & aller & Guinée & pendant trois semaines \\
\hline & & & allé & & en Guinée & pendant trois semaines \\
\hline
\end{tabular}

29 On trouve beaucoup d'alternances de ce type dans les données du discours bilingue fongbe-français. Nous les traitons comme des phénomènes d'alternance dans la mesure où les structures internes sont rigoureusement conformes à celle de la langue française. Il semble qu'il $\mathrm{y}$ a une distribution complémentaire entre les positions où il $\mathrm{y}$ a alternance et celles où on a des emprunts.

\subsubsection{Alternances inter-phrastiques}

On peut en observer quelques exemples dans les données suivantes :

\begin{tabular}{|l|l|l|l|l|l|l|l|l|l|}
\hline (4) & a. & & yè & nà & kánbyò & wè & & & $\begin{array}{l}\text { si tu indisposes les } \\
\text { gens }\end{array}$ \\
\hline & & on & FUT & demander & toi & & & & \\
\hline & 'On & te le & demandera & si tu & $\begin{array}{l}\text { les } \\
\text { gens' }\end{array}$ & & & \\
\hline & & & & & & & & \\
\hline & b. & hwè & dòkpá & dò & fidé & ó & & & c'est plus calme \\
\hline & & toi & un & être & lieu & TOP & & & \\
\hline & & 'Quand & tu vis & seul, & c'est & plus & & & calme' \\
\hline & & & & & & & & \\
\hline & c. & à & mò & $\grave{c}$ & dò & scène & jí & o & il a de la peine à se \\
déplacer
\end{tabular}




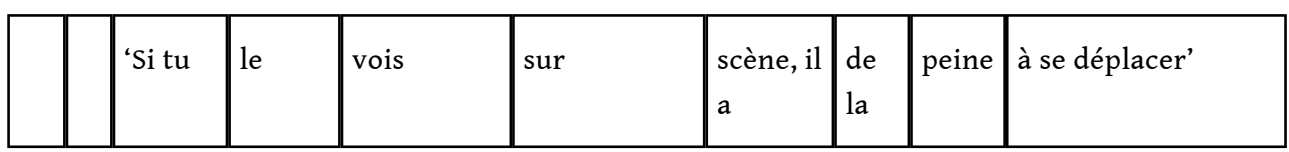

\subsection{Alternances extra-phrastiques}

Les alternances extra-phrastiques sont balisées, et sont constitués essentiellement de répétitions, d'hésitations et de formes figées comme : "quoi", "disons" "parce que", etc.

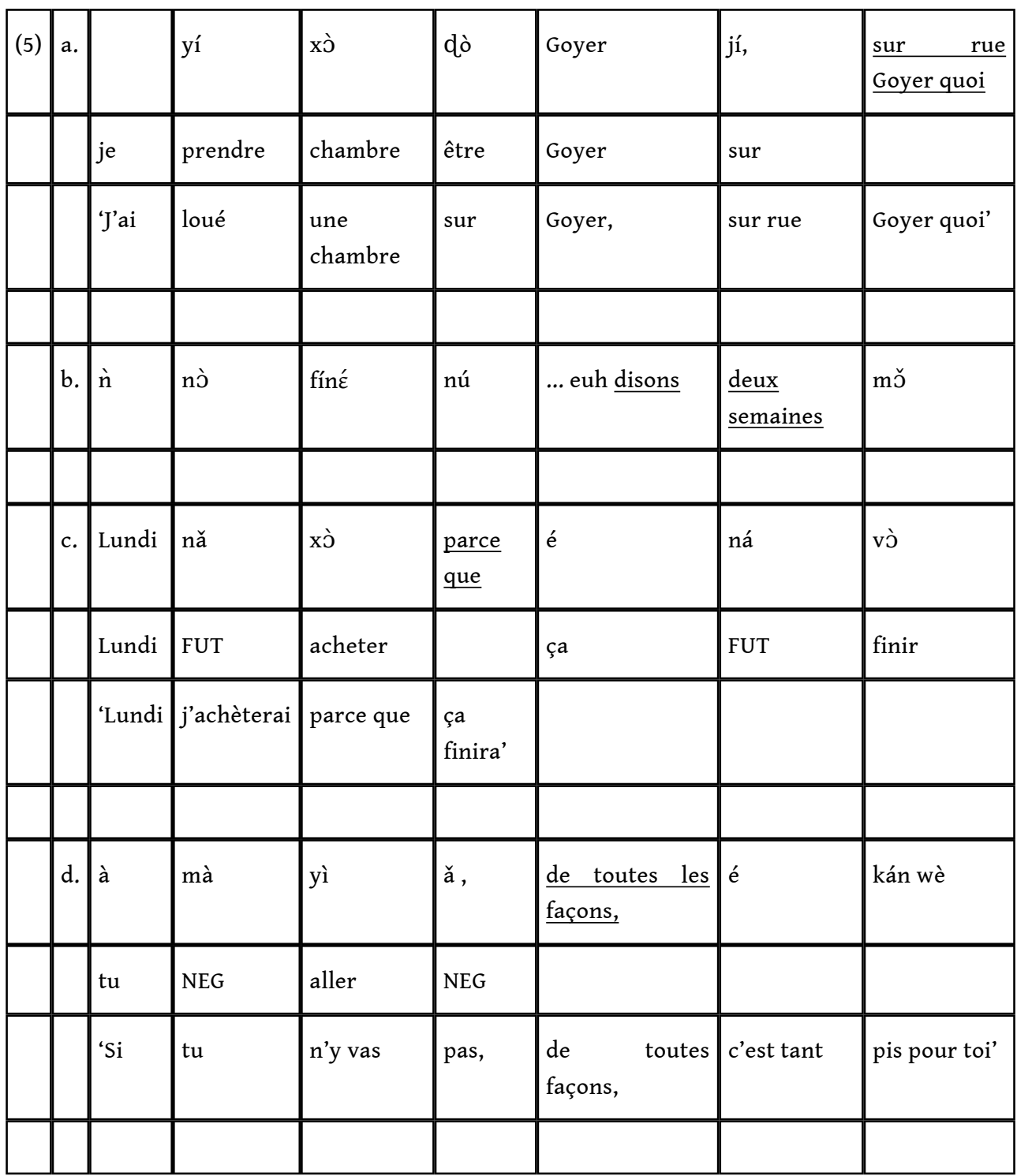

\section{Emprunt de lexèmes verbaux}

Dans cette section nous étudions le comportement des lexèmes verbaux d'origine française dans le parler des bilingues fongbe-français. Il s'agira de savoir à quel type de faits de mélange nous avons affaire. Nous verrons que les lexèmes verbaux sont en 
majorité des emprunts d'origine française. Pour des raisons méthodologiques, nous distinguerons le comportement des verbes à la forme perfective de ceux à la forme imperfective.

\subsection{Les verbes à la forme perfective}

Le fongbe est une langue qui se caractérise par une absence totale de flexion sur les verbes contrairement au français où tous les verbes sont fléchis dans les énoncés. Le fongbe fonctionne avec un système de modes et d'aspects marqués par des morphèmes qui n'ont aucune influence sur la morphologie du verbe. Il est donc intéressant de voir comment les verbes français sont traités quand ils entrent dans le parler des bilingues.

On observe des occurrences de lexèmes verbaux du français dans les données suivantes :

\begin{tabular}{|c|c|c|c|c|c|c|c|c|}
\hline (6) & a. & yè & nó & dépanner & $m \varepsilon \grave{~}$ & l⿳亠े & & \\
\hline & & ils & HAB & dépanner & gens & PLU & & \\
\hline & & 'Ils & & dépannent & les gens' & & & \\
\hline & b. & à & nă & orienter & yě & & & \\
\hline & & tu & FUT & orienter & eux & & & \\
\hline & & ‘tu & les & orienteras' & & & & \\
\hline & c. & nyé & j̀, & $\grave{n}$ & nò & suivre & mode & ă \\
\hline & & moi & TOP & je & HAB & suivre & mode & NEG \\
\hline & & 'Quant & à & moi, je ne & suis & pas la & mode' & \\
\hline
\end{tabular}

La question est de savoir si nous avons affaire à des emprunts spontanés ou bien à des alternances.

En nous référant à Pfaff (1979), nous pouvons dire que si le fongbe était une langue flexionnelle, la procédure la plus simple serait d'observer la flexion prise par le verbe. Si cette flexion est celle du fongbe on en déduira que le verbe est un emprunt du français. Mais si cette flexion est celle du français, alors il s'agira d'une alternance. Mais nous ne sommes pas dans une telle situation. L'observation des données nous permettra quand même de distinguer les emprunts des alternances. En effet, dans la plupart des cas, les verbes français dans le parler des bilingues sont à la forme infinitive du français. Il est parfois difficile de savoir s'il s'agit d'un infinitif ou d'une autre forme, comme un participe passé par exemple, et c'est particulièrement vrai pour les verbes du premier groupe (terminaison en er). Dans un énoncé comme (7) ci-dessous, 


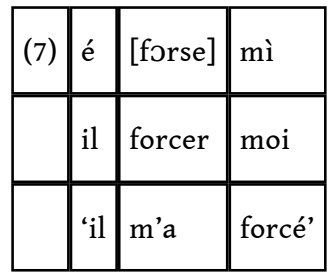

rien ne nous indique si le verbe est forcer ou forcé. Cependant quand on prend des données comportant des verbes du troisième groupe, on voit bien que la forme du verbe est l'infinitif, ce qu'on peut constater dans les énoncés suivants :

\begin{tabular}{|l|l|l|l|l|l|l|l|}
\hline (8) & a. & éyè & ná & l'́́v & affranchir & sédó & wè \\
\hline & & lui & FUT & ITER & affranchir & envoyer & toi \\
\hline & & 'Lui, & il te & l'affranchira & de nouveau' & & \\
\hline & & & & & & & \\
\hline & b. & yě & & descendre & bò yì & dó & \\
\hline & & ils & & descendre & et aller & en bas & \\
\hline & & 'Ils sont & & descendus & et sont & allés en bas' & \\
\hline
\end{tabular}

Cela est conforme à la situation observée dans beaucoup de données de mélanges de langues. Par exemple, Sankoff et al. (1986) ont noté ce fait pour le tamoul, et Kachru (1978) pour le hindi, face à l'anglais : d'une manière générale, les verbes sont empruntés sous leur forme infinitive et sont précédés ou suivis (selon le cas) de divers marqueurs de la langue emprunteuse indiquant des catégories grammaticales comme l'aspect, le temps, le mode etc. C'est précisément le cas pour le fongbe. Une analyse quantitative des données fait apparaître que sur les verbes du troisième groupe contenus dans ces données, $95 \%$ sont à la forme infinitive.

\subsection{Un cas d'alternance de verbe}

Il y a donc $5 \%$ des verbes qui ne sont pas à la forme infinitive. On peut en observer un exemple en (9) ci-dessous :

\begin{tabular}{|l|l|l|l|l|l|l|l|}
\hline$(9)$ & é & dépend & lě & à & & dègbòn & ò \\
\hline & Ça & dépend & comment & tu & & être & TOP \\
\hline \hline & 'Ça' & dépend & de comment & tu & es' & & \\
\hline
\end{tabular}

Comme on le voit dans cet énoncé, le verbe n'est pas à la forme infinitive, et il porte une flexion provenant de la langue française. Par ailleurs, chez l'un des locuteurs de notre 
corpus, on observe que cette forme est précédée d'une réplique complètement en français :

\begin{tabular}{|l|l|l|l|l|l|l|}
\hline (10) & Ça dépend... & é & dépend & hwènù & wè & ó \\
\hline & Ça dépend.... & ça & dépend & moment & c'est & TOP \\
\hline & Ça dépend,.... & 'ça & dépend & du moment & où & c'est' \\
\hline
\end{tabular}

Il s'agit sans aucun doute d'un fait de balisage. Nous pouvons en déduire que dans ce dernier énoncé, il s'agit d'une alternance. D'une manière générale, on peut donc dire que, dans le parler des bilingues fongbe-français, les verbes qui sont à la forme infinitive relèvent du phénomène d'emprunt, alors que les autres relèvent de l'alternance. Cette conclusion est corroborée par l'étude de Meechan \& Poplack (1995), qui montre que les participes passés des verbes français qui sont introduits en fongbe, et qui ne sont donc pas à la forme infinitive, sont des alternances et non des emprunts.

\subsection{Les verbes à la forme imperfective}

41 La forme des verbes dans les constructions imperfectives est une preuve complémentaire que la plupart des verbes sont des emprunts dans le parler des bilingues fongbe-français.

42 L'imperfectif des verbes intransitifs s'exprime en fongbe par une construction comportant une réduplication de la première syllabe du verbe, comme on le voit dans les exemples suivants :

\begin{tabular}{|l|l|}
\hline (11) & Perfectif \\
\hline & Kòjó wá \\
\hline & K. venir \\
\hline & 'Kodjo est venu' \\
\hline & \\
\hline b. & Kòjó sísò \\
\hline & K. trembler \\
\hline & 'Kodjo a tremblé' \\
\hline & \\
\hline$(12)$ & Imperfectif \\
\hline & \\
\hline a. & Kòjó dó wìwá wè \\
\hline
\end{tabular}




\begin{tabular}{|l|l|}
\hline & K. être venir PROG \\
\hline & 'Kodjo est en train de venir' \\
\hline & \\
\hline b. & Kòjó dó sísísó wè \\
\hline & K. être trembler PROG \\
\hline & 'Kodjo est en train de trembler' \\
\hline
\end{tabular}

43 Ces phénomènes ont été étudiés dans Tossa (1994). Sur le plan sémantique, la forme imperfective correspond à ce qu'on appelle le "progressif", qu'on trouve par exemple en anglais dans une phrase comme "john is crying". La réduplication est un phénomène morphologique du fongbe, qui correspond à peu près au gérondif.

Dans le parler des bilingues fongbe-français, on remarque que les verbes du français qui entrent dans cette construction subissent la même réduplication.

\begin{tabular}{|c|c|c|}
\hline (13) & a. & secteur éné l'́ \\
\hline & & secteur DEM PLU qui commencer créer PROG \\
\hline & & 'Ces secteurs qui ont commencé à se créer...' \\
\hline & b. & ...type ó dò cri-critiquer wè \\
\hline & & ...type DET être critiquer PROG \\
\hline & & '...le type est en train de critiquer' \\
\hline & c. & 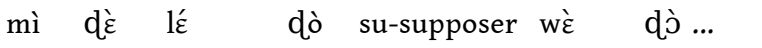 \\
\hline & & nous REF PLU être supposer \\
\hline & & 'Nous autres sommes en train de supposer que ...' \\
\hline
\end{tabular}

On voit que le verbe a subi une modification morphologique conforme à la structure du fongbe. Il s'agit donc là d'un emprunt spontané au français.

\section{Pronom clitique et emprunt}

Tim (1975), Wentz \& McClure (1976) et Pfaff (1979) ont postulé une contrainte qui stipule que les pronoms objets clitiques sont toujours réalisés dans la même langue que les verbes auxquels ils sont attachés et dans la position exigée par les règles syntaxiques de la 
langue de l'élément qui reçoit le clitique. Il nous faut indiquer que dans certaines langues, les clitiques objets sont des morphèmes qui se cliticisent sur le verbe et parfois font subir une flexion à ce verbe. C'est le cas en fongbe. Dans une telle situation, la contrainte dont il est question ici n'est pas fondamentalement différente de la contrainte du morphème libre proposée par Poplack puisque ces pronoms doivent être considérés comme des morphème liés quand ils sont cliticisés sur le verbe. Woolford (1983), pour illustrer cette contrainte, propose les exemples suivants :

\begin{tabular}{|l|l|l|}
\hline (14) & a. & Yo lo compré \\
\hline & b. & *Yo lo bought \\
\hline & c. & *I/Yo it compré \\
\hline & d. & *I/Yo it bought \\
\hline & & 'I bought it.' \\
\hline
\end{tabular}

47 Le pronom lo est un clitique en espagnol. La règle syntaxique qui génère les pronoms objets clitiques exige que ce pronom soit en position préverbale en espagnol. Ce qui n'est pas le cas en anglais, d'où l'agrammaticalité des exemples (14b, c, d).

Dans le parler bilingue fongbe-français, on observe un phénomène singulier où, pour échapper à la contrainte du pronom objet clitique, les locuteurs utilisent une stratégie qui se présente comme une sorte de recatégorisation d'un morphème.

Observons les énoncés en (15) ci dessous :

\begin{tabular}{|l|l|l|l|}
\hline (15) & a. & Allô ton papa est là ?... & Passer tòn nú mì \\
\hline & & Allô ton papa est là ?... & Passer POSS PREP moi \\
\hline & & 'Allô ton papa est là ?... & Passe-le moi.' \\
\hline & & & \\
\hline & b. à nǎ sǐn & nourrir tòn, soigner tòn, \\
\hline & & tu FUT pouvoir & nourrir POSS soigner \\
& & & POSS \\
\hline & & 'Tu pourras & le nourrir, le soigner.' \\
\hline
\end{tabular}

L'énoncé en (15a) est un extrait de dialogue téléphonique où un bilingue fongbe-français demande à parler au père de l'enfant qui a décroché le combiné du téléphone. L'énoncé "Passer tòn nú mì » comporte plusieurs "anomalies" grammaticales qui méritent une étude particulière.

51 On peut observer ici que le verbe est à la forme infinitive. Le bilingue prononce phonétiquement [pase] au lieu de [pas]. Cette forme contraste avec la forme impérative 
que le verbe aurait en français. On peut donc admettre qu'à ce niveau il s'agit d'un emprunt.

Mais le bilingue utilise la particule tòn pour exprimer le pronom objet de 3e personne exprimé par le en français. En fongbe, cette particule ne fonctionne jamais comme pronom objet. La particule tòn est un marqueur de possessif, comme on le voit dans l'exemple suivant :

\begin{tabular}{|l|l|l|l|}
\hline$(16)$ & àvǔn & póvlù & tòn \\
\hline & chien & Paul & POSS \\
\hline & 'Le chien & de Paul' & \\
\hline
\end{tabular}

53 La particule tòn joue aussi le rôle de pronom possessif à la troisième personne du singulier. On a :

\begin{tabular}{|l|l|l|}
\hline$(17)$ & cè & le mien \\
\hline & tòwè & le tien \\
\hline & é tòn & le sien \\
\hline
\end{tabular}

54 En fongbe, le pronom objet est toujours une voyelle qui est cliticisée sur le verbe. Sa forme morphophonologique dépend de la dernière voyelle du verbe. Pour un verbe se terminant par [e], le pronom objet clitique doit être [e] comme dans :

\begin{tabular}{|l|l|l|l|l|}
\hline (18) & ž̃ & è & nú & mì \\
\hline & prendre & le & PREP & moi \\
\hline & 'Prends-le moi' & & & \\
\hline
\end{tabular}

Donc on devrait s'attendre à une forme comme "Passer è nú mi" (passe le moi). Mais une forme telle que celle-là ne se trouve jamais dans le langage du bilingue fongbe-francais.

La contrainte du clitique exigerait que le locuteur dise "Passer le nú mi". Mais il fait face ici à deux exigences apparemment contradictoires : changer de langue à ce niveau précis entre le verbe et son complément, et respecter en même temps la contrainte du clitique. Nous postulons que la solution qu'il a trouvé est d'aller chercher dans le stock des morphèmes disponibles un candidat susceptible de remplir la même fonction que le clitique [è] mais qui n'est pas le clitique lui-même. L'élément qui possède les traits susceptibles de remplir cette fonction, à savoir les traits de pronom et de troisième personne, est le morphème tòn. Ce morphème a donc subi une recatégorisation qui le fait passer de marqueur de possessif au statut de pronom objet. 
57 Au total la portion de discours en (15a) ; "Allô ton Papa est là ?...Passer tòn nú mì"comporte une alternance inter-phrastique du français vers le fongbe où la frontière se situe juste avant le verbe passer. Une pause sert de balisage à cette alternance. Le changement de langue a lieu à partir de l'emprunt spontané du verbe passer.

Nous voyons que la contrainte du morphème libre proposée par Poplack (ou celle du pronom objet clitique) se vérifie empiriquement avec les données des bilingues fongbefrançais. Mais le résultat le plus intéressant est que les bilingues peuvent être amenés à des stratégies diverses en utilisant les ressources internes du système de leur langue pour éviter les contraintes relatives au mélange de langues (ici celle du morphème libre). La stratégie utilisée est la recatégorisation du morphème tòn.

Notre analyse confirme aussi le fait qu'un emprunt spontané n'a pas exactement le même statut qu'un emprunt intégré. Si les deux sortes d'emprunt avaient le même statut, la cliticisation du [è] aurait dû se faire sur le verbe, car le locuteur aurait considéré "passer" comme un verbe normal du fongbe. Il faut donc se demander pourquoi une forme telle que "Passer è nú mi" n'est jamais attestée chez le bilingue. Notre hypothèse est que, d'une certaine manière, les locuteurs bilingues continuent de percevoir cet emprunt spontané comme un lexème de la deuxième langue. Les emprunts spontanés se trouveraient comme sur un "no man's land" où ils sont candidats à une intégration complète dans la deuxième langue. C'est ce que fait remarquer Romaine (1995 : 59) quand elle écrit que les items nouvellement empruntés semblent avoir un statut linguistique incertain, pendant quelques temps, avant d'être complètement intégrés. Poplack, Sankoff et Miller (1988) ont aussi remarqué que la variabilité dans l'assignation des genres aux mots empruntés décroît dès que la fréquence de l'utilisation du mot emprunté s'accroît. Selon nous, cette variabilité est une preuve que les emprunts spontanés ont un statut incertain. Quand la fréquence d'utilisation s'accroît, le mot passe progressivement de son statut d'emprunt spontané au statut d'emprunt intégré.

Il est donc prévisible que la forme "Passer è nú mi", c'est-à-dire la forme avec le clitique en fon, soit tôt ou tard attestée chez les bilingues fongbe-français.

\section{Emprunt d'un constituant : le verbe et son complément}

61 Dans le parler des bilingues fongbe-français on observe des énoncés comme ceux en (19) ci-dessous ${ }^{3}$ :

\begin{tabular}{|l|l|lll|}
\hline$(19)$ & a. & é poser question $\varnothing \quad$ nú pólù \\
\hline & & il poser question INDEF PREP Paul \\
\hline & & 'Il a posé une question à Paul' \\
\hline & & & \\
\hline & b. & ǹ nò suivre mode $\varnothing$ \\
\hline & & je HAB suivre mode INDEF \\
\hline
\end{tabular}




\begin{tabular}{|l|l|l|}
\hline & & \multicolumn{2}{l|}{ 'Je suis la mode' } \\
\hline & & \\
\hline & c. & à nă percer civilisation dé \\
\hline & & tu FUT percer civilisation INDEF \\
\hline & & 'Tu perceras une (certaine) civilisation' \\
\hline
\end{tabular}

En présence de données comme celles-là, il faut savoir si on à affaire à une alternance ou bien à un autre type de mélange de langue.

La plupart des théories syntaxiques reconnaissent que le verbe et son complément forment un constituant. Quand on se réfère à la théorie X-barre, par exemple (Chomsky (1982)), la structure qu'il faut donner au syntagme verbal dans un énoncé comme (19a) cidessus est la suivante :

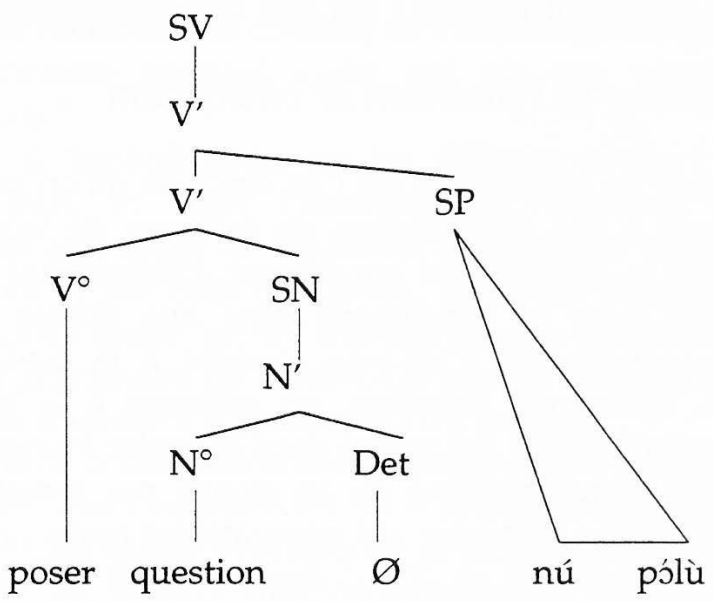

Dans (20), le syntagme verbal est constitué de deux autres syntagmes intermédiaires dont l'un est [V' poser question Ø]. C'est ce syntagme qui constitue la portion de phrase en français. Sommes-nous en présence d'une alternance, d'un emprunt, ou d'un autre fait de contact de langues?

Pour déterminer le fait de mélange auquel nous avons affaire ici, nous considérons chacun des éléments que sont le verbe d'une part et son complément d'autre part. Dans les exemples en (19), les verbes peuvent être analysés comme étant à l'infinitif, eu égard à la forme suivre obtenue dans (19b) par contraste avec la forme fléchie qu'il a en français : les verbes sont des emprunts. En ce qui concerne les compléments, on peut remarquer qu'ils sont intégrés syntaxiquement parce que leurs déterminants sont en fongbe. Donc les compléments sont aussi des emprunts. Nous sommes alors en présence d'un emprunt spontané simultané du verbe et de son complément. Il s'agit d'un emprunt de tout un constituant. Les éléments entrant dans la composition de ce constituant qui est ici un V' ont été, de façon interne, réarrangés pour servir comme emprunt spontané. Il apparaît que la différence typologique entre les deux langues est responsable de ce réarrangement. L'absence de flexion sur les verbes en fongbe et la différence de position des marqueurs des verbes forment une configuration où la contrainte d'équivalence entre 
en jeu. Par ailleurs, au niveau du syntagme nominal objet, la même contrainte empêche l'alternance entre le nom et le déterminant, d'où l'emprunt du nom.

En conclusion, les données du parler bilingue fongbe-français nous permettent de dire que dans les phénomènes de mélange de langues, l'emprunt spontané ne concerne pas seulement les unités minimales, mais qu'il peut porter aussi sur des unités plus grandes. Cette forme d'emprunt spontané, à notre connaissance, n'a pas encore été relevée dans la littérature, et elle diffère du phénomène d'insertion de constituant dont nous allons parler dans la section suivante.

\section{Insertion de constituant : un cas de resubordination}

\subsection{Le phénomène de l'insertion de constituant}

67 L'insertion de constituant est l'un des faits qu'on peut rencontrer quand on est confronté à des phénomènes de mélange de langues. Elle consiste à introduire dans le discours un constituant grammatical d'une langue en un point approprié pour ce constituant dans une phrase d'une autre langue. L'insertion de constituant n'est pas soumise à la contrainte d'équivalence. Généralement, après une insertion, on retourne à la langue de base. Naït M’Barek \& Sankoff (1988) ont relevé dans le parler bilingue arabe-français des cas de mélange intraphrastique où on trouve par exemple un nom plus un déterminant d'origine française qui apparaissent après un démonstratif arabe, comme dans les exemples en (21) ci-dessous (Naït M’Barek \& Sankoff (1988 : 149)) :

\begin{tabular}{|l|l|l|}
\hline (21) & a. & Le charme walla hadik la particularité dyal les Clubs Meds. \\
\hline & & ou cette de \\
\hline & & 'Le charme ou bien la particularité des Clubs Meds.' \\
\hline & & qu' ils t'ont donné cette \\
\hline \hline & & beureusement lli ctawak had la bourse \\
\hline & & 'Heureusement qu'ils t'ont donné cette bourse.' \\
\hline & & c. bhal duk les avions légers... \\
\hline & & miši n'est pas comme ces \\
\hline & & 'Ce n'est pas comme les avions légers...' \\
\hline
\end{tabular}

On observe, en outre, des cas où un nom plus un déterminant français apparaissent après un déterminant arabe, en l'occurrence, le prédéterminant wahed qui sémantiquement correspond à un indéfini, comme on peut le voir dans les exemples suivants (Naït M'Barek \& Sankoff (1988: 150)) : 


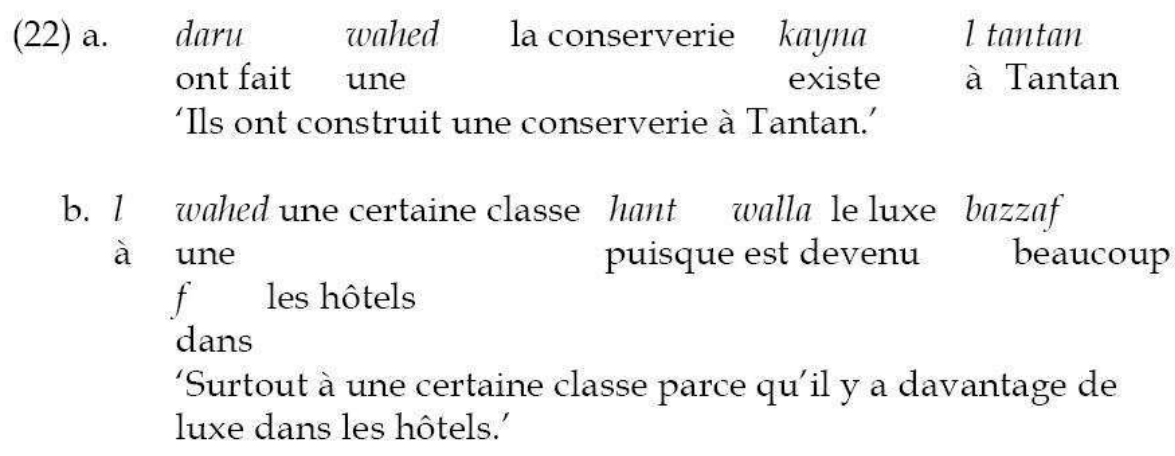

69 Comme l'indiquent Naït M'Barek \& Sankoff, ces structures, démonstratif + SN et défini + $\mathrm{SN}$, sont propres à l'arabe et non au français. Ils analysent ces faits comme n'étant ni des alternances ni des emprunts, mais plutôt comme des "insertions du groupe nominal, qui consiste en l'utilisation du groupe nominal français dans un contexte nominal arabe qui n'a pas nécessairement de contrepartie en français."

Naït M'Barek \& Sankoff précisent les différences entre l'insertion et l'alternance d'une part, et entre l'insertion et l'emprunt d'autre part. L'alternance se réalise aux types de frontières syntaxiques engendrées par la grammaire du français et par celle de l'arabe, alors que l'insertion consiste à utiliser, dans un contexte arabe, un SN français dont la structure interne n'est pas contrainte par la grammaire de l'arabe. De plus la position du SN dans la phrase arabe n'est pas soumise à la grammaire du français.

71 En ce qui concerne la distinction entre l'emprunt et l'insertion du SN, ces deux auteurs indiquent que l'insertion du SN n'implique que des fragments de phrases qui respectent les règles syntaxiques et morphologiques du français; tandis que l'emprunt est intégré morphologiquement et syntaxiquement à l'arabe.

Naït M'Barek \& Sankoff font remarquer en outre que l'emprunt affecte des mots simples, des mots composés ou de courtes expressions figées, tandis que l'insertion implique aux moins deux mots, un article et un nom. Nous allons voir que l'insertion peut porter sur un seul mot.

Un autre cas d'insertion de constituant a été relevé par Poplack \& Sankoff (1988: 1179) dans le discours bilingue tamoul-anglais. En tamoul, les compléments phrastiques sont généralement terminés par une particule quotative $n u$ suivie par un verbe selon le schéma suivant: Phrase $+n u+V^{2} e^{4}$ correspondant à peu près à la structure anglaise Verbe + that + Phrase. Poplack \& Sankoff indiquent qu'on observe fréquemment dans le discours bilingue tamoul-anglais la structure : Phrase anglaise $+n u+$ Verbe tamoul. Cela ne peut être dû à une alternance de langue, à cause de la contrainte d'équivalence. Ils concluent qu'il s'agit d'un cas d'insertion de constituant.

Nous sommes donc là en présence de deux cas différents d'insertion de constituant : l'un concernant un syntagme nominal, et l'autre une phrase entière. Nous allons montrer que dans les données du discours bilingue fongbe-français, il y a un cas d'insertion d'un mot: le subordonnant que.

\subsection{Une re-subordination : l'insertion du subordonnant que}

75 On a relevé dans certaines langues du monde que la forme morphologique du verbe signifiant "dire" est la même que celle de la particule qui représente le subordonnant que (cf. : Lord 1976; Koopman 1984). En fongbe, on observe des données qui peuvent faire 
penser à une utilisation du verbe j̀ "dire" comme subordonnant que. On peut l'observer dans les exemples en (23) ci-dessous :

\begin{tabular}{|c|c|c|c|c|c|c|c|}
\hline (23) & a. & é & mó & dj̀ & vì & ó & yàvì \\
\hline & & il & voir & dire/que & enfant & DEF & pleurer \\
\hline & & ‘Il a & $\mathrm{vu}$ & que & l'enfant & a pleuré' & \\
\hline & b. & é & & dj̀ & pólù & wá & \\
\hline & & il & croire & dire/que & Paul & venir & \\
\hline & & ‘Il & a cru & que & Paul & est venu' & \\
\hline & c. & é & dò & dj̀ & pólù & wá & \\
\hline & & il & dire & dire/que & Paul & venir & \\
\hline & & ‘Il & a dit & que & Paul & est venu' & \\
\hline
\end{tabular}

Dans Tossa (1994), nous avons montré que le verbe j̀ "dire" ne correspond pas à un subordonnant de type que comme on pourrait le croire (cf. aussi Kinyalolo 1992). En fait ce verbe entre dans une construction à série verbale, c'est-à-dire une structure syntaxique dans laquelle deux ou plusieurs lexèmes verbaux apparaissent dans une phrase (ou une proposition), en succession pour exprimer un seul événement ou bien une séquence d'événements. Le fongbe fait une large utilisation de ces types de construction. Dans les énoncés en (23) ci-dessus, le verbe "dire" n'est pas le subordonnant que. Il est plutôt dans une structure de sérialisation où il occupe la position du deuxième verbe de la série. La structure que nous postulons pour ces énoncés est (24) ci-dessous, avec une illustration par l'exemple (23c).

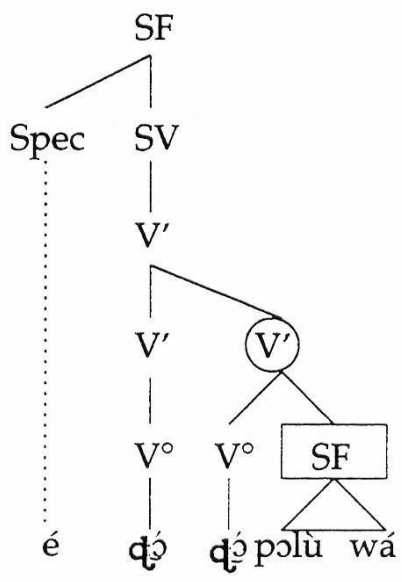


Il s'agit d'une configuration où le deuxième verbe "dire" (celui qui correspond à que) est dans un syntagme verbal V' (entouré d'un cercle dans la représentation en arbre) qui est en adjonction (en termes de X-barre) à un autre V'. En fait le fongbe ne dispose pas de subordonnant de type que. Cette langue compense l'absence de ce type de subordonnant par l'utilisation de la sérialisation verbale dans laquelle intervient le verbe signifiant "dire". En fongbe, ce verbe ne peut prendre en complément qu'un syntagme fonctionnel SF.

Dans le discours des bilingues fongbe-français, on observe des données comme celles en (25) ci-dessous :

\begin{tabular}{|l|l|l|l|l|l|l|l|l|l|l|}
\hline$(25)$ & a. & nyè & mò & ò & & que langue & ò & é & dò & importante \\
\hline & & moi & voir & que & & langue & DET & il & être & importante \\
\hline & & & & & & & & & & \\
\hline & b. & ǹ & dò & certain & dò & que & àz ò & dé & lé & dè \\
\hline & & je & être & certain & que & travail & INDEF & & PLU & être \\
\hline & & 'Je & suis & certain & qu & 'il & y & a & certains & travaux' \\
\hline & & & & & & & & & & \\
\hline
\end{tabular}

79 Le fait qui nous intéresse ici est la présence du que dans ces énoncés, qui n'est indispensable ni pour la grammaticalité ni pour le sens de ces énoncés. Il s'agit simplement, de la part des bilingues, d'un effet de style dont les fondements sont plutôt d'ordre pragmatique. Il y a cependant une justification syntaxique à la présence du que dans ces énoncés. C'est ce que nous allons analyser.

L'analyse que nous proposons est qu'il s'agit d'une insertion de constituant, ici un constituant ultime. Nous postulons que le bilingue fait une "vraie" subordination selon la structure suivante :

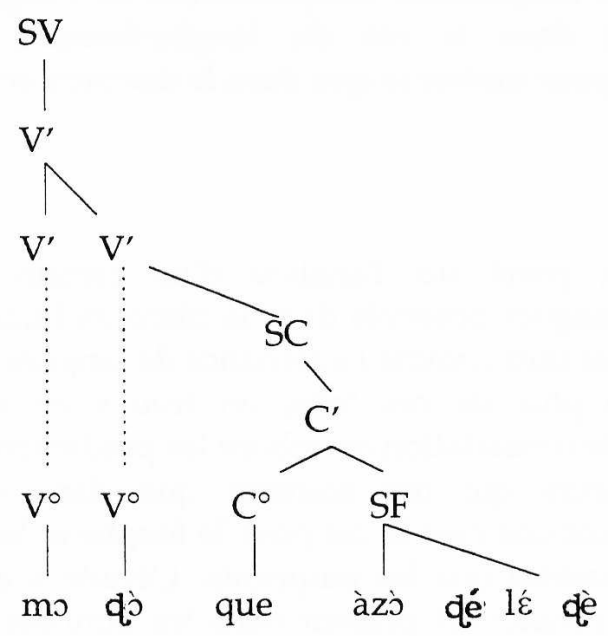


passe comme si le bilingue remplaçait le constituant de type SF par un constituant de type SC. Ce faisant il dispose d'une position syntaxique (qui est ici $\mathrm{C}^{\circ}$ ) pour insérer le que. Tandis que dans le discours monolingue fongbe le verbe $\grave{j}$ (en deuxième position dans (24)) ne peut prendre qu'un complément de type SF, les bilingues fongbe-français imposent à ce verbe un complément de type SC.

Avec une telle analyse, on voit bien qu'il s'agit d'une insertion rendue possible grâce à une restructuration de la phrase opérée par le bilingue. Il fait une re-subordination conformément à la structure de la langue française qui exige le subordonnant que. Le phénomène d'insertion de constituant reçoit de ce fait une explication syntaxique rigoureuse.

L'intérêt que présentent les données du discours bilingue fongbe-français est que dans le phénomène d'insertion de constituant, le constituant inséré peut être un constituant ultime.

Des cas de mélange de langues portant sur la présence d'un subordonnant de type que d'une deuxième langue dans une première ont déjà été notés empiriquement. Sankoff \& Poplack (1981: 34) cite l'exemple (27) ci-dessous dans le discours bilingue espagnol portoricain-anglais.

\begin{tabular}{|l||l|}
\hline (27) & I could understand que you don't know how to speak Spanish, \\
\hline & verdad. \\
\hline & 'Je pourrais comprendre que tu ne sais pas comment parler \\
\hline & espagnol, n'est-ce pas.' \\
\hline
\end{tabular}

Woolford (1983) considère qu'il s'agit d'une alternance à la frontière entre le subordonnant et le reste de la proposition. Dans le cas du discours bilingue fongbe français, il ne pourrait s'agir d'une alternance puisque cette configuration violerait la contrainte d'équivalence du point de vue du fongbe. Il s'agit en fait de deux processus différents: dans le cas du parler bilingue portoricain-anglais, il y a eu simplement remplacement de l'anglais that par l'espagnol que, alors que dans le cas du fongbefrançais il y a eu restructuration de la phrase pour insérer le que dans le discours en fongbe.

\section{Conclusion}

La présente étude a porté sur l'analyse d'un certain nombre de phénomènes de contact de langues observés dans le discours bilingue fongbe-français. Elle a montré que des faits comme l'alternance de langues sont attestés dans ce discours. Mais en plus de ces faits, on trouve en majorité des phénomènes d'emprunt. Cette constatation corrobore les prédictions théoriques faites par d'autres chercheurs qui ont soutenu que dans des langues typologiquement éloignées (comme c'est le cas pour le fongbe et le français) les alternances sont moins fréquentes que les emprunts. L'étude a aussi montré qu'un autre phénomène de contact est présent dans les données. Il s'agit du phénomène d'insertion de constituant. Nous avons identifié deux formes particulières de ce phénomène et nous 
leur avons proposé des analyses originales qui sont une contribution à la connaissance de ce phénomène.

\section{BIBLIOGRAPHIE}

BENTAHILA, A., DAVIES, E. (1983) “The syntax of Arabic-French code-switching” Lingua, 59, 301-330.

воКАмВА, Е. G. (1988) “Code-mixing, language variation, and linguistic theory : Evidence from Bantu languages," Lingua, 76, 21-62.

Сномsку, N. (1982) Some Concepts and Consequences of the Theory of Government and Binding, Cambridge Mass. : MIT Press.

GARDNER-CHLOROS P. (1983) “Code-switching : Approches principales et perspectives”, La linguistique, vol. 19, fasc. 2, 21-53.

GOBBI, E. (1990) Studio della commutazione di codice in situazione di diglossia, Maîtrise de Psycholinguistique, Université de Rome.

GUMPERZ J. J. (1976) “The sociolinguistic significance of conversational code-switching," in J. Cook-Gumperz \& J. J. Gumperz (Eds.), Papers on language and context, University of California Working Papers 46, Berkeley : University of California.

KACHRU, B. (1978) “Toward structuring code-mixing : an Indian perspective”, International Journal of the Sociology of Language 16, 28-46.

KINYALOLO, K.K.W. (1992) “The Status of ò in Fon”, in : C. Lefebvre \& J. Lumsden (dir.), La genèse du créole haitien : un cas particulier d'investigation sur la forme de la grammaire universelle, Rapport de recherche pour l'année 1991-1992, Université du Québec à Montréal, pp. 59-82.

KOOPMAN, H. (1984) The Syntax of Verbs, Dordrecht : Foris.

LABOV, W. (1969) "Contraction, deletion and inherent variability of the English copula" Language 45 (4), 715-762.

Labov, W. (1971) “Some principles of linguistic methodology" Language in Society, 1, 97-120.

LABOV, W. (1984) "Field methods of the project on linguistic change and variation," in Baugh, J. and Sherzer, J. (éds.) Language in use, Englewood Cliffs, New-Jersey : Prentice-Hall.

LORD, C. (1976) "Evidence for syntactic Reanalysis : From Verb to Complementizer in Kwa", in : Sanford, B. Steever ; Carol, A. Walker \& Salikoko, J. Mufwene (eds.), Papers from the Parassession on Diachronic Syntax, Chicago Linguistic Society, pp. 179-191.

MAHOOTIAN, S. ; B., Santorini (1996) “Code Switching and Complement / Adjunct Distinction”, Linguistic Inquiry, 27 (3), 464-479.

MEECHAN, M. ; S. POPLACK (1995) "Orphan categories in bilingual discourse : Adjectivization strategies in Wolof-French and Fongbe-French" Language Variation and Change, 7 (2), 169-194.

MEILLET, A. (1921) Linguistique historique et linguistique générale, Paris, Société Linguistique de Paris. 
NAÏT M’BAREK, M. \& D., SANKOFF (1988) “Le discours mixte arabe/français : des emprunts ou des alternances de langue ?", Canadian Journal of Linguistics /Revue canadienne de linguistique, 33, 143-154.

PFAFF, C. (1979) “Constraints on language mixing”, Language, 55, 291-318.

POPLACK, S. (1980) “Sometimes I'll start a sentence in Spanish y termino en español : Toward a typology of code-switching", Linguistics 18, 581-618.

POPLACK, S. (1981) "Syntactic structure and social function of code-switching”, in : R. Duran (ed.) Latino discourse and communicative behavior, New Jersey : Ablex, pp. 169-184.

POPLACK, S. (1982) "Bilingualism and the vernacular," in : B. Hartford, A.Valdman and C. Foster (Eds.), Issues in international bilingual education: The role of the vernacular, New York : Plenum Press, pp. 1-23.

POPLACK, S. (1988) "Conséquences linguistiques du contact de langues : un modèle d'analyse variationniste" Langage et société, 43, 23-48.

POPLACK,. S. (1989) "Statut de langue et accomodation langagière le long d'une frontière linguistique", in : R. Mougeon et E. Beniak (eds.) Le français canadien parlé hors Québec : aperçu sociolinguistique, Québec : Presses de l'Université Laval, pp. 127-151.

POPLACK S. (1993) "Variation theory and language contact" in D. Preston (ed.), American dialect research : an anthology celebrating the 100th anniversary of the American dialect society, Amsterdam : John Benjamins, pp. 251-286.

POPLACK S., D., SANKoff (1988) “Code-Switching”, in : U. Ammon, N. Dittmar, K. Mattheir (ed) Sociolinguistics; An international handbook of the science of language and society; Berlin :Walter de Gruyter, 1174-1180.

POPLACK, S., D., SANKOFF \& C. MILLER (1988) "The social correlates and linguistic consequences of lexical borrowing and assimilation", Linguistics 26, 47-104.

POPLACK, S. ; WHEELER, D. \& WESTWOOD A. (1987) “Distinguishing language contact phenomena : evidence from Finnish-English bilingualism”, in : P. Lilius \& M. Saari (eds.) The Nordic language and Modern Linguistics 6, Helsinki : University of Helsinki Press.

REYES, R. (1974) Studies in Chicano Spanish, Harvard University, Cambridge, MA. (Thèse de Ph. D.) ROMAINE S. (1995) Bilingualism, Oxford, Blackwell.

SANKOFF, D. (1982) “Sociolinguistic method and Linguistic theory” in L. Cohen et al. (eds.), Logic, Methodology, Philosophy of SciencesVI, Amsterdam : North Holland, pp. 679-687.

SANKOFF, D. (1988) “Sociolinguistics and syntactic variation”, in : F. Newmeyer (ed.), Linguistics : the Cambridge Survey, New York : Cambridge University Press, pp. 140-161.

SANKOFF, D. \& S. POPLACK (1981) “A formal grammar for code-switching”, Papers in Linguistics : International Journal of Human Communication 14, 3-45.

SANKOFF, D. ; POPLACK S. \& S. Vanniarajan (1986) The case of the Nonce Loan in Tamil, Centre de recherches mathématiques appliquées, Rapport technique 1348, Université de Montréal.

SANKOFF, G. (1974) "A quantitative paradigm for the study of communicative competence", in : R. Bauman and J. Sherzer (Eds.) Exploration in Ethnography of Speaking, New York : Academic Press, pp. 1-36.

SAPIR, E. (1921) Language, New York : Harcourt \& Brace. 
SILVESTRI, E. (1996) L'alternance des langues dans une conversation familiale bilingue italocanadienne, Thèse de Doctorat, Université René Descartes-Paris V.

TITONE, R. (1994) “Some Italian investigations of 'code switching' in diglossic settings : a summary report" La linguistique 30 (2), 65-73.

TOSSA C. Z. (1994) Adjonction et séries verbales dans les langues gbe. Thèse de Ph.D. Université d'Ottawa.

TIM, L. (1975) “Spanish-English Code-switching : el porque y how-not-to”, Romance Philology 28, 473-482.

WENTZ, J. \& E. MCCLURE (1976) “Ellipsis in Bilingual Discourse”, in: S. Mufwene, C. Walker \& S. Steever (eds.), Papers from the Twelfth Regional Meeting of the Chicago Linguistics Society, University of Chicago, Chicago, Illinois, 656-665.

WOOLFORD, E. (1983) "Bilingual Code-switching and Syntactic Theory", Linguistic Inquiry 14 (3), 520-536.

\section{NOTES}

1. La recherche ayant conduit à cette publication a été effectuée lors d'un séjour au Département des Sciences du Langage de l'Université de Paris 10 Nanterre grâce à une subvention accordée par l'AUPELF-UREF. Nous présentons ici nos remerciements au Professeur Colette Noyau, Directrice du Département, pour ses conseils, ses suggestions et son soutien moral et matériel.

2. Haugen (1956), cité par Gardner-Chloros (1983)

3. En fongbe l'indéfini est un morphème zéro. Cette langue distingue l'indéfini absolu caractérisé par le morphème zéro, l'indéfini relatif marqué par le morphème é, et le défini marqué par Ov.

4. Le tamoul est une langue à structure SOV.

\section{RÉSUMÉS}

La présente étude analyse le discours bilingue fongbe-français pratiqué notamment à Cotonou (Bénin) en dégageant les caractéristiques formelles de certains phénomènes de contact qui y interviennent.

Après avoir présenté le cadre théorique dans lequel s'insèrent les analyses, nous étudions le phénomène d'alternance ou code-switching, puis les phénomènes d'emprunt, qui sosnt majoritaires (confirmant par là la position selon laquelle dans des couples de langues typolo giquement éloignées les alternances sont moins fréquentes que les emprunts), ceux d'insertion de constituant, et nous analysons un cas particulier de ce phénomène aboutissant à la restructuration syntaxique de la phrase affectée.

Our study analyses Fongbe-French bilingual discourse as spoken in Benin, and especially in Cotonou, and highlights the formal features of some contact phenomena which characterize it. After presenting our theoretical frame, we analyze the main contact phenomena: codeswitching, then borrowing - which is most frequent, thus confirming the position that in 
typologically very different language pairs, borrowing by far outweighs code-switching, and finally constituent insertion, offering a detailed account of one such case, which leads to sentence restructuring.

\section{AUTEUR}

\section{COMLAN-ZÉPHIRIN TOSSA}

Université Nationale du Bénin 